\title{
Qualidade de vida das gestantes de baixo risco de Teresina - PI
}

\author{
Quality of life of low risk pregnant women in Teresina - PI
}

Calidad de vida de los gestantes de bajo riesgo de Teresina - PI

\begin{abstract}
Ane Kássia de Carvalho Barbosa ${ }^{1 *}$, Bruna Furtado Sena de Queiroz¹, Dannyel Roger Almeida Teixeira1, Esaú de Castro Mourão², Gabriela Mayner Silva Cantanhede1, Kamille Regina Costa de Carvalho', Karla Manuela Sobral Batista1', Letícia Maria Carvalho Sousa ${ }^{3}$, Lívia Florêncio de Brito', Lorena Magalhães Chaves ${ }^{1}$ Matheus Henrique da Silva Lemos ${ }^{1}$, Natasha Louise Silva Ribeiro ${ }^{1}$, Sara Ferrão Jacques ${ }^{1}$, Roberta Fortes Santiago ${ }^{4}$, Taciany Alves Batista Lemos ${ }^{1}$.
\end{abstract}

\section{RESUMO}

Objetivo: avaliar a qualidade de vida das gestantes de baixo risco. Métodos: pesquisa quantitativa, descritiva e transversal realizada com 250 gestantes através de questionário sociodemográfico e Índice de Qualidade de Vida de Ferrans \& Powers. Resultados: as gestantes tinham entre 19 e 24 anos, parceiro fixo, pardas, religião católica, renda média mensal de 1 a 2 salários, desempregada, $2^{\circ}$ grau completo; já tem filho, sem história de abortos, sem planejamento gestacional e intercorrências gestacionais. Predominou as gestantes que iniciaram o pré-natal no $1^{\circ}$ trimestre. Verificou-se que a maioria não apresentou nenhum vício. A Qualidade de Vida é boa, no qual o domínio socioeconômico obteve maiores escores e o domínio psicológico/espiritual os menores. Obteve-se associação significativa na idade, no domínio psicológico/espiritual; escolaridade, renda e trabalho nos domínios saúde/funcionamento e psicológico/espiritual; número de filhos no domínio socioeconômico; abortos no domínio família e saúde/funcionamento; planejamento gestacional e início do pré-natal nos domínios saúde/funcionamento e socioeconômico. Conclusão: acredita-se que esta pesquisa possa contribuir para a busca de melhorias na qualidade dos cuidados prestados as gestantes de baixo risco, com a finalidade de se elevar ainda mais a qualidade de vida dessas gestantes.

Palavras-chave: Qualidade de vida, Gestação, Saúde da mulher.

\begin{abstract}
Objective: to evaluate the quality of life of low-risk pregnant women. Methods: quantitative, descriptive and cross-sectional study with 250 pregnant women through a sociodemographic and IQVFP questionnaire. Results: pregnant women were between 19 and 24 years old, a fixed partner, browns, catholic religion, average monthly income of 1 to 2 salaries, unemployed, 2 nd grade; already has a child, no history of abortions, no gestational planning and gestational intercurrences. Pregnant women who started prenatal care in the first trimester prevailed. It was verified that the majority did not present any vice. QoL is good, in which the socioeconomic domain obtained higher scores and the psychological / spiritual domain the lower. Significant association was obtained in age, in the psychological / spiritual domain; schooling, income and work in the health / functioning and psychological / spiritual domains; number of children in the socioeconomic domain; abortions in the family and health / functioning domains; gestational planning and prenatal initiation in the
\end{abstract}

\footnotetext{
${ }^{1}$ Faculdade Integral Diferencial (FACID), Teresina-PI. *E-mail: ane.kassia@hotmail.com

${ }^{2}$ Cristo Faculdade do Piauí (CHRISFAPI), Piripiri-PI

3Universidade Estadual do Piauí (UESPI), Teresina-PI

${ }^{4}$ Universidade Federal do Piauí (UFPI), Teresina-PI
} 
health / functioning and socioeconomic domains. Conclusion: it is believed that this research may contribute to the search for improvements in the quality of care provided to low-risk pregnant women, in order to raise the quality of life of these pregnant women even more.

Keywords: Quality of life, Pregnancy, Women's health.

\section{RESUMEN}

Objetivo: evaluar la calidad de vida de las gestantes de bajo riesgo. Métodos: investigación cuantitativa, descriptiva y transversal realizada con 250 gestantes a través de cuestionario sociodemográfico e IQVFP. Resultados: las gestantes tenían entre 19 y 24 años, socio fijo, pardas, religión católica, renta media mensual de 1 a 2 salarios, desempleada, $2^{\circ}$ grado completo; ya tiene hijo, sin historia de abortos, sin planificación gestacional e intercurrencias gestacionales. Predominó a las gestantes que iniciaron el prenatal en el primer trimestre. Se verificó que la mayoría no presentó ningún vicio. La QV es buena, en la cual el dominio socioeconómico obtuvo mayores escores y el dominio psicológico / espiritual los menores. Se obtuvo una asociación significativa en la edad, en el dominio psicológico / espiritual; escolaridad, renta y trabajo en los ámbitos salud / funcionamiento y psicológico / espiritual; número de hijos en el dominio socioeconómico; abortos en el ámbito familiar y salud / funcionamiento; la planificación gestacional y el inicio del prenatal en los ámbitos salud / funcionamiento y socioeconómico. Conclusión: se cree que esta investigación puede contribuir a la búsqueda de mejoras en la calidad de los cuidados prestados a las gestantes de bajo riesgo, con la finalidad de elevar aún más la calidad de vida de esas gestantes.

Descriptores: Calidad de vida, Gestación, Salud de la mujer.

\section{INTRODUÇÃO}

A assistência pré-natal faz-se de grande importância e deve estar voltada as necessidades da gestante nos âmbitos físico, psíquico e sociocultural, sendo esses aspectos fundamentais para garantia de qualidade de vida durante a gestação e puerpério. A qualidade de vida frente ao ciclo gravídico-puerperal é efetivamente consolidada quando a mulher adquire conhecimentos e informações pertinentes ao pré-natal, de modo a apreender o nível de qualidade de vida que pode vivenciar de maneira mais segura, direcionada e individual (BEZERRA IFD, et al., 2015).

Ferrans e Powers definem a Qualidade de Vida (QV) como a percepção subjetiva do nível de felicidade e de satisfação em relação aos diferentes aspectos da vida, sendo que esse conjunto de variáveis que contempla a definição proposta pode influenciá-la negativamente e/ou positivamente. Ou seja, transcende o estado biológico, condição de saúde psicológica e social e ao se abordar a QV em gestante, envolve ainda a própria qualidade do atendimento prestado à mulher no âmbito do ciclo gravídico-puerperal, abrangendo desde a quantidade mínima de consultas, ao inicio do pré-natal, bem como exames e vacinas necessárias (TIRADO MCBA, et al., 2014).Ou

Existem alguns instrumentos os quais vêm sendo utilizados para mensurar a QV de gestantes, dentre eles tem o Ferrans and Powers Quality of Life Índex. Tais instrumentos se fundamentam em sólida base conceitual e metodológica, o que justifica a sua ampla aceitação como instrumento de avaliação subjetiva da qualidade de vida, em diversos países (OLIVEIRA AS e SANTOS VLC de G, 2011).

$\mathrm{Na}$ área da saúde existe uma oportunidade de atuação do enfermeiro como agente educador que, para empoderar, precisa conhecer a sua população e contexto. Identificar essas características é uma forma de indicar ações necessárias para que o parto e o nascimento sejam ancorados nos direitos humanos das mulheres que são atendidas no sistema público de saúde (SILVA MC et al., 2018).

Com a finalidade de contribuir para as pesquisas sobre a qualidade de vida de gestantes de baixo risco e de conhecer a realidade do município de Teresina, PI, justifica-se este estudo. Portanto, é importante conhecer a qualidade de vida de sua clientela para buscar sua melhoria mediante atividades promotoras de 
saúde. Assim, surge a seguinte problemática: Qual a qualidade de vida das gestantes de baixo risco cadastradas em equipes de Estratégia Saúde da Família no município de Teresina - PI?

A escolha da temática deu-se em virtude da escassez de pesquisas que avaliam a qualidade de vida aplicando o Índice de Ferrans \& Powers ao contexto do ciclo gravídico-puerperal, especialmente voltadas às gestantes de baixo risco.

Para solucionar tal indagação, traçou-se o seguinte objetivo geral: avaliar a qualidade de vida das gestantes de baixo risco cadastradas em equipes de Estratégia Saúde da Família no município de TeresinaPI. Como objetivos específicos: caracterizar a amostra por meio das variáveis sociodemográficas, obstétricas e quanto a hábitos de vida; analisar a qualidade de vida de gestantes utilizando o instrumento de Qualidade de vida de Ferrans \& Powers adaptado; e comparar a qualidade de vida das gestantes com as variáveis sociodemográficas, obstétricas e hábitos de vida.

\section{MÉTODOS}

A pesquisa realizou-se com submissão prévia ao Termo de Consentimento Institucional (TCI), bem como pela autorização das participantes do estudo, através da assinatura do Termo de Consentimento Livre e Esclarecido (TCLE) ou Termo de Assentimento Livre e Esclarecido (TALE), em caso de gestantes com idade inferior a 18 anos.

Trata-se de um estudo prospectivo, quantitativo, do tipo descritivo-exploratório e de corte transversal. A pesquisa teve como cenário as Unidades Básicas de Saúde (UBS) vinculadas a Estratégia Saúde da Família (ESF) do município de Teresina, incluídas no estudo através de escolha aleatória de forma não intencional mediada pelo programa Project for Statistical Computing (Versão 3.0.2).

As participantes foram 250 gestantes que usufruíram do atendimento pré-natal de baixo risco vinculado à Estratégia Saúde da Família do município de Teresina - PI. Como critérios para inclusão das participantes foram adotados: gestantes que usufruíam do atendimento pré-natal de baixo risco vinculado a Estratégia Saúde da Família do município de Teresina - PI e que concordaram em participar da pesquisa. Como critérios de exclusão: gestantes com atendimento pré-natal de alto risco e/ou gestantes com atendimento pré-natal de baixo risco que apresentasse intercorrências individuais e relacionadas à gestação que alterassem a condição de baixo risco a alto risco, no que se refere ao atendimento pré-natal prestado; bem como gestantes que não concordaram em assinar o TCLE ou TALE.

As entrevistas foram realizadas em Unidades Básicas de Saúde do município de Teresina-PI, totalizando 12 locais de atendimento de saúde. As entrevistas eram feitas a partir de abordagem individual das gestantes após a realização de consultas pré-natal ou participação em grupos de atividade coletiva. Dentre os instrumentos utilizados citam-se: o questionário com perguntas relacionadas a dados sociodemográficos, obstétricos e de hábitos de vida das gestantes. Em seguida aplicou-se o instrumento Índice de Qualidade de Vida de Ferrans \& Powers adaptado (IQVFP). A aplicação dos questionários era realizada pela graduanda. Após coleta, os dados foram submetidos ao tratamento estatístico pelo programa SPSS, versão 20.0. Para apresentação dos resultados utilizou-se tabelas e gráficos.

A pesquisa obedeceu à Resolução 466/12 do Conselho Nacional em Saúde. O estudo foi submetido à avaliação e aprovado pela Comissão de Ética da Fundação Municipal de Saúde e da Faculdade Integral Diferencial, recebendo autorização para início da pesquisa através do número de parecer: 1.530.468.

\section{RESULTADOS E DISCUSSÃO}

\section{Perfil das gestantes em atendimento pré-natal de baixo risco}

Observa-se pela Tabela 1 que participaram do estudo gestantes com idade entre 14 e 34 anos, predominando a faixa etária de 19 a 24 anos $(56,4 \%)$, com idade média de 23,34 anos. Frequências semelhantes também foram encontradas em estudo realizado no Piauí, com faixa etária que variou entre os limites 14 e 35 anos (LIMA IFA, 2015). 
Nos demais dados sociodemográficos verifica-se que a maioria se autodeclararam pardas $(50,4 \%)$, têm a religião católica $(78 \%)$, apresentam o $2^{\circ}$ grau completo $(65,6 \%)$, têm parceiro $(88,4 \%)$, usufruem de um a dois salários mínimos $(48,4 \%)$, sendo relevante a porcentagem de gestantes que sobrevivem com menos de um salário mínimo (45,6\%). Ressalte-se que a maior parte das participantes são desempregadas $(53,6 \%)$. Em estudo regional, realizado em Teresina, verifica-se a predominância de gestantes que possuem o 20 grau completo $41,2 \%$ (NUNES PBS, 2015).

Tabela 1 - Distribuição do perfil sociodemográfico das gestantes que usufruem do atendimento pré-natal de baixo risco vinculado a Estratégia Saúde da Família, Teresina - PI, 2018.

\begin{tabular}{|c|c|c|}
\hline Variáveis & $\mathbf{N}$ & $\%$ \\
\hline \multicolumn{3}{|l|}{ Faixa Etária } \\
\hline$<19$ anos & 17 & 6,8 \\
\hline $19-24$ anos & 141 & 56,4 \\
\hline $25-29$ anos & 80 & 32,0 \\
\hline$>29$ anos & 12 & 4,8 \\
\hline Mínimo - Máximo & \multirow{3}{*}{\multicolumn{2}{|c|}{$\begin{array}{c}14-34 \\
23,34 \pm 3,76 \\
22,87-23,81\end{array}$}} \\
\hline Médio — Desvio Padrão & & \\
\hline IC95\% & & \\
\hline \multicolumn{3}{|l|}{ Cor } \\
\hline Branca & 65 & 26,0 \\
\hline Parda & 126 & 50,4 \\
\hline Negra & 59 & 23,6 \\
\hline \multicolumn{3}{|l|}{ Religião } \\
\hline Católica & 195 & 78,0 \\
\hline Evangélica & 45 & 18,0 \\
\hline Outras & 10 & 4,0 \\
\hline \multicolumn{3}{|l|}{ Escolaridade } \\
\hline Analfabeta & - & - \\
\hline $1^{\circ}$ grau incompleto & - & - \\
\hline $1^{\circ}$ grau completo & 11 & 4,4 \\
\hline $2^{\circ}$ grau incompleto & 74 & 29,6 \\
\hline $2^{\circ}$ grau completo & 164 & 65,6 \\
\hline Superior incompleto & 1 &, 4 \\
\hline \multirow{2}{*}{\multicolumn{3}{|c|}{ Renda }} \\
\hline & & \\
\hline Menos de um salário mínimo & 114 & 45,6 \\
\hline De um a dois salários mínimos & 121 & 48,4 \\
\hline De dois a três salários mínimos & 15 & 6,0 \\
\hline Três ou mais salários mínimos & - & - \\
\hline \multicolumn{3}{|l|}{ Trabalho } \\
\hline Sim & 116 & 46,4 \\
\hline Não & 134 & 53,6 \\
\hline \multicolumn{3}{|l|}{ Parceiro } \\
\hline Sim & 221 & 88,4 \\
\hline Não & 29 & 11,6 \\
\hline Total & 250 & 100,0 \\
\hline
\end{tabular}

Legenda: N, frequência absoluta; \%, frequência relativa com IC 95\%.

Fonte: Barbosa AKC, Queiroz BFS, Teixeira DRA, et al., 2019.

Ao se avaliar a Tabela 2, percebe-se pelos dados obstétricos que a maior parte das gestantes já tem um filho (42\%), não têm história de abortos anteriores (89,6\%); não planejaram a gestação $(84,4 \%)$, e não tiveram intercorrências gestacionais $(96,4 \%)$. No que se refere ao período gestacional vigente, $49,6 \%$ estavam no $1^{\circ}$ trimestre, $34 \%$ no $2^{\circ}$ trimestre e $16,4 \%$ no $3^{\circ}$ trimestre. É importante mencionar que predominou as que iniciaram o pré-natal no $1^{\circ}$ trimestre de gestação $(84,8 \%)$. Quanto aos hábitos de vida durante a gestação, verifica-se que $95,6 \%$ não apresentam nenhum vício; das que apresentam vícios, destaca-se as tabagistas $(4,4 \%)$. 
Tabela 2 - Distribuição da condição de saúde das gestantes que usufruem do atendimento pré-natal de baixo risco vinculado a Estratégia Saúde da Família, Teresina - PI, 2018.

\begin{tabular}{|c|c|c|}
\hline Variáveis & $\mathbf{N}$ & $\%$ \\
\hline \multicolumn{3}{|l|}{ Número de filhos vivos } \\
\hline Um & 105 & 42,0 \\
\hline Dois & 71 & 28,4 \\
\hline Três & 21 & 8,4 \\
\hline Quatro & 2 & ,8 \\
\hline Nenhum & 51 & 20,4 \\
\hline \multicolumn{3}{|l|}{ Abortos anteriores } \\
\hline $\operatorname{Sim}$ & 26 & 10,4 \\
\hline Não & 224 & 89,6 \\
\hline \multicolumn{3}{|l|}{ Período gestacional } \\
\hline $1^{\circ}$ trimestre & 124 & 49,6 \\
\hline $2^{\circ}$ trimestre & 85 & 34,0 \\
\hline $3^{\circ}$ trimestre & 41 & 16,4 \\
\hline \multicolumn{3}{|l|}{ Planejamento da gravidez } \\
\hline Sim & 39 & 15,6 \\
\hline Não & 211 & 84,4 \\
\hline \multicolumn{3}{|l|}{ Início do pré-natal } \\
\hline $1^{\circ}$ trimestre & 212 & 84,8 \\
\hline $2^{\circ}$ trimestre & 38 & 15,2 \\
\hline $3^{\circ}$ trimestre & - & - \\
\hline \multicolumn{3}{|c|}{ Intercorrências durante gestação } \\
\hline $\operatorname{Sim}$ & 9 & 3,6 \\
\hline Não & 241 & 96,4 \\
\hline \multicolumn{3}{|l|}{ Hábitos de vida gestação } \\
\hline Tabagismo & 11 & 4,4 \\
\hline Elitismo & - & - \\
\hline Consumo de drogas ilícitas & - & - \\
\hline Nenhum & 239 & 95,6 \\
\hline Total & 250 & 100,0 \\
\hline
\end{tabular}

Legenda: $\mathrm{N}$, frequência absoluta; \%, frequência relativa com IC 95\%.

Fonte: Barbosa AKC, Queiroz BFS, Teixeira DRA, et al., 2019.

Em estudo realizado em Campina Grande, $92,5 \%$ das mulheres iniciaram o pré-natal antes da $20^{\underline{a}}$ semana de gravidez, não havendo dificuldades na atenção precoce das gestantes e retardamento nas consultas. ${ }^{7}$ Em relação ao início do pré-natal, em estudo realizado em Teresina, $73,4 \%$ das gestantes iniciaram o pré-natal no $1^{\circ}$ trimestre, assim como é recomendado pelo MS (BRASIL, 2012).

\section{Qualidade de vida das gestantes de baixo risco}

A Tabela 3 revelou que QV geral das gestantes de baixo risco teve como média o escore de 23,99. Nessa perspectiva, o domínio que obteve maior pontuação foi o domínio Socioeconômico com uma média de 27,28, enquanto o domínio que teve menor pontuação foi o Psicológico/espiritual com a média de 21,04. 
Tabela 3 - Qualidade de Vida para cada domínio do instrumento de Ferrans \& Powers adaptado das gestantes que usufruem do atendimento pré-natal de baixo risco vinculado a Estratégia Saúde da Família, Teresina - PI, 2018.

\begin{tabular}{lcccc}
\hline \hline Domínios & Mínimo & Máximo & Média & Desvio padrão \\
\hline Geral & 0,00 & 30,00 & 23,99 & 7,09 \\
Saúde/funcionamento & 0,00 & 30,00 & 23,42 & 6,76 \\
Socioeconômico & 5,00 & 30,00 & 27,28 & 4,41 \\
Psicológico/espiritual & 0,00 & 30,00 & 21,04 & 8,77 \\
Família & 2,50 & 30,00 & 27,22 & 3,96 \\
\hline \hline
\end{tabular}

Fonte: Barbosa AKC, Queiroz BFS, Teixeira DRA, et al., 2019.

Escore geral de QV semelhante foi encontrado em estudo com gestantes cardiopatas, onde o escore total foi de 23,9 (MENEGUIN e XAVIER, 2013).

\section{Relação da qualidade de vida das gestantes com os dados sociodemográficos e obstétricos}

Para relacionar a QV das gestantes de baixo risco com os dados socioeconômicos e obstétricos obtidos foram utilizados o teste $U$ de Mann-Whitney quando a comparação se tratava de dois grupos independentes e o teste Krusskall-Wallis para comparação de mais de dois grupos com a finalidade de detectar associações significativas entre a média dos escores em cada um dos domínios do questionário IQVFP adaptado, considerando um nível de significância de 5\%, ou seja, com 95\% de confiabilidade.

Como observado na Tabela 4, no que se refere à idade das gestantes desse estudo houve associação significativa com a QV no domínio psicológico/espiritual, evidenciando uma relação direta com a idade $(p<0,001)$. Assim, verifica-se que gestantes adolescentes apresentam uma menor qualidade de vida no domínio psicológico/espiritual, quando comparada a idades mais elevadas. Acredita-se que o achado de menor QV no domínio psicológico/espiritual em gestantes adolescentes possa ser explicado pelo comprometimento dos aspectos que envolvem tal domínio, dentre eles, paz de espírito, fé em Deus, objetivos pessoais, felicidade, satisfação com a vida, aparência pessoal e consigo própria.

Tabela 4 - Domínio do Índice de Qualidade de Vida de Ferrans \& Powers adaptado em relação à idade das gestantes que usufruem do atendimento pré-natal de baixo risco vinculado a Estratégia Saúde da Família, Teresina - PI, 2018.

\begin{tabular}{|c|c|c|c|c|c|}
\hline Domínio & Idade & $\mathbf{N}$ & Média & Desvio padrão & p-valor \\
\hline \multirow[t]{4}{*}{ Saúde/funcionamento } & $<19$ anos & 17 & 22,62 & 1,26 & $0,056^{*}$ \\
\hline & $19-24$ anos & 141 & 23,44 & 1,19 & \\
\hline & $25-29$ anos & 80 & 23,52 & 1,25 & \\
\hline & $>29$ anos & 12 & 23,54 & 1,31 & \\
\hline \multirow[t]{4}{*}{ Psicológico/espiritual } & $<19$ anos & 17 & 18,57 & 1,68 & $<0,001^{*}$ \\
\hline & $19-24$ anos & 141 & 20,79 & 2,64 & \\
\hline & $25-29$ anos & 80 & 21,80 & 2,20 & \\
\hline & $>29$ anos & 12 & 22,41 & 2,63 & \\
\hline \multirow[t]{4}{*}{ Socioeconômico } & $<19$ anos & 17 & 28,67 & 1,77 & $0,085^{\star}$ \\
\hline & $19-24$ anos & 141 & 27,01 & 2,82 & \\
\hline & $25-29$ anos & 80 & 27,40 & 2,48 & \\
\hline & $>29$ anos & 12 & 26,40 & 3,02 & \\
\hline \multirow[t]{4}{*}{ Família } & $<19$ anos & 17 & 27,38 & 2,15 & $0,715^{\star}$ \\
\hline & $19-24$ anos & 141 & 27,08 & 2,44 & \\
\hline & 25 - 29 anos & 80 & 27,56 & 1,80 & \\
\hline & $>29$ anos & 12 & 27,66 & 1,81 & \\
\hline
\end{tabular}

Legenda: *Teste $\mathrm{H}$ de Kruskal Walis.

Fonte: Barbosa AKC, Queiroz BFS, Teixeira DRA, et al., 2019. 
Como observado na Tabela 5, no que se refere à escolaridade das gestantes, houve associação significativa com a QV nos domínios saúde/funcionamento e psicológico/espiritual $(p<0,001)$, onde se verifica uma relação direta com a idade. Em estudo realizado com gestantes também se verificou uma associação significativa da escolaridade com a QV no domínio psicológico/espiritual ( $p=0,037)$. Entretanto, a relação foi inversamente proporcional, onde indivíduos com menor nível de escolaridade apresentaram maior QV nesse domínio (NUNES PBS, 2015).

Tabela 5 - Domínio do Índice de Qualidade de Vida de Ferrans \& Powers adaptado em relação à escolaridade das gestantes que usufruem do atendimento pré-natal de baixo risco vinculado a Estratégia Saúde da Família, Teresina - PI, 2018.

\begin{tabular}{|c|c|c|c|c|c|}
\hline Domínios & Escolaridade & $\mathbf{N}$ & Média & Desvio padrão & p-valor \\
\hline \multirow[t]{4}{*}{ Saúde/funcionamento } & $1^{\circ}$ grau completo & 11 & 22,48 & 1,37 & \multirow[t]{4}{*}{$<0,001^{\star}$} \\
\hline & $2^{\circ}$ grau incompleto & 74 & 23,01 & 1,17 & \\
\hline & $2^{\circ}$ grau completo & 164 & 23,65 & 1,18 & \\
\hline & Superior incompleto & 1 & 25,34 & & \\
\hline \multirow[t]{4}{*}{ Psicológico/espiritual } & $1^{\circ}$ grau completo & 11 & 19,58 & 1,78 & \multirow[t]{4}{*}{$<0,001^{*}$} \\
\hline & $2^{\circ}$ grau incompleto & 74 & 19,21 & 2,09 & \\
\hline & $2^{\circ}$ grau completo & 164 & 21,92 & 2,30 & \\
\hline & Superior incompleto & 1 & 28,06 & - & \\
\hline \multirow{4}{*}{ Socioeconômico } & 1ㅇ grau completo & 11 & 26,84 & 2,75 & \multirow{4}{*}{0,182} \\
\hline & $2^{\circ}$ grau incompleto & 74 & 26,78 & 2,90 & \\
\hline & $2^{\circ}$ grau completo & 164 & 27,46 & 2,57 & \\
\hline & Superior incompleto & 1 & 23,86 & - & \\
\hline \multirow[t]{4}{*}{ Família } & $1^{\circ}$ grau completo & 11 & 27,36 & 2,03 & \multirow{4}{*}{0,815} \\
\hline & $2^{\circ}$ grau incompleto & 74 & 27,19 & 2,19 & \\
\hline & $2^{2}$ grau completo & 164 & 27,32 & 2,24 & \\
\hline & Superior incompleto & 1 & 26,25 & - & \\
\hline
\end{tabular}

Legenda: *Teste $\mathrm{H}$ de Kruskal Walis

Fonte: Barbosa AKC, Queiroz BFS, Teixeira DRA, et al., 2019.

Como observado na Tabela 6, no que se refere à renda das gestantes, houve associação significativa com a QV nos domínios saúde/funcionamento e psicológico/espiritual $(p<0,001)$, onde se verifica uma relação direta com a renda.

Tabela 6 - Domínio do Índice de Qualidade de Vida de Ferrans \& Powers adaptado em relação à renda das gestantes que usufruem do atendimento pré-natal de baixo risco vinculado a Estratégia Saúde da Família, Teresina - PI, 2018.

\begin{tabular}{llrrrr}
\hline \hline Domínio & Renda & N & Média & Desvio padrão & p-valor \\
\hline Saúde/funcionamento & Menos de um salário mínimo & 114 & 22,96 & 1,17 & $<\mathbf{0 0 1}^{*}$ \\
& De um a dois salários mínimos & 121 & 23,79 & 1,17 & \\
& De dois a três salários mínimos & 15 & 23,85 & 1,07 & \multirow{2}{*}{ Psicológico/espiritual } \\
& Menos de um salário mínimo & 114 & 19,32 & 1,95 & $<\mathbf{0 0 1}^{*}$ \\
& De um a dois salários mínimos & 121 & 22,34 & 2,04 & \\
Socioeconômico & De dois a três salários mínimos & 15 & 23,64 & 2,51 & \multirow{2}{*}{$0,268^{*}$} \\
& Menos de um salário mínimo & 114 & 27,04 & 2,88 & \\
Família & De um a dois salários mínimos & 121 & 27,46 & 2,54 & \\
& De dois a três salários mínimos & 15 & 26,66 & 2,34 & \multirow{2}{*}{$0,724^{*}$} \\
& Menos de um salário mínimo & 114 & 27,31 & 2,34 & \\
\hline \hline
\end{tabular}

Legenda: *Teste $\mathrm{H}$ de Kruskal Walis.

Fonte: Barbosa AKC, Queiroz BFS, Teixeira DRA, et al., 2019. 
Estudo realizado em São Paulo com gestantes de risco habitual converge com tais achados ao apontar significância na relação entre o vínculo empregatício e os domínios saúde/funcionamento e psicológico/espiritual, de forma diretamente proporcional (FERREIRA FM, et al, 2013).

Não houve associação significativa da QV em nenhum domínio considerando o período gestacional, presença de intercorrência e de vícios. Estudos realizados em São Paulo também encontraram associação similar da QV com o planejamento da gravidez (MENEGUIN e XAVIER, 2013; MAÇOLA L, et al, 2010).

\section{CONCLUSÃO}

O estudo permitiu verificar que a QV das gestantes de baixo risco é relativamente boa, no qual o domínio socioeconômico obteve maiores escores e o domínio psicológico/espiritual os menores escores. Dessa forma é importante ressaltar a grande relevância deste estudo, o qual se faz inédito, por evidenciar a QV de gestantes de baixo risco em Teresina-PI. Acredita-se que esta pesquisa possa contribuir para a busca de melhorias na qualidade dos cuidados prestados as gestantes.

\section{REFERÊNCIAS}

1. BEZERRA IFD, et al. Comparação da qualidade de vida em gestantes com disfunção sexual. Revista Brasileira de Ginecologia Obstetrícia, 2015; 37(6): 266-271.

2. FERREIRA FM, et al. Qualidade de vida de adolescentes após maternidade. Revista Acta Paulista Enfermagem, 2013; 26(3): 245-9.

3. LIMA IFA. Pré-natal em Teresina: análise da assistência segundo as recomendações do programa de humanização de pré-natal e nascimento [monografia]. Faculdade Integral Diferencial - FACID|Wyden, Teresina, 2015.

4. MENEGUIN S, XAVIER C. Qualidade de vida em gestantes com cardiopatia. Revista Texto Contexto Enfermagem, 2013; 22(3): 811-8.

5. MAÇOLA L, et al. Avaliação da autoestima de gestantes com uso da Escala de Autoestima de Rosenberg*. Revista da Escola de Enfermagem USP, 2010; 44(3): 570-7.

6. MINISTÉRIO DA SAÚDE (BR), Secretaria de Atenção a Saúde. Departamento de Atenção Básica. Atenção ao prénatal de baixo risco [Internet]. Brasília: Editora do Ministério da Saúde; 2012. (Cadernos de Atenção Básica, 32).

7. NUNES PBS. Qualidade de vida das gestantes de alto risco acompanhadas em um centro de referência [monografia]. Universidade Estadual do Piauí - UESPI, Teresina, 2015.

8. OLIVEIRA AS, SANTOS VLC de G. Responsividade dos instrumentos de avaliação de qualidade de vida de Ferrans \& Powers: uma revisão bibliográfica. Acta Paulista Enfermagem, 2011; 24(6): 839-44.

9. PEDRAZA DF, et al. Assistência pré-natal e peso ao nascer: uma análise no contexto de unidades básicas de saúde da família. Revista Brasileira de Ginecologia Obstetrícia, 2013; 35(8): 349-56.

10. SILVA MC, et al. Parto e nascimento na região rural: a violência obstétrica. Revista de Enfermagem UFPE, $2018 ; 12$ (9): 2407-17.

11. TIRADO MCBA, et al. Qualidade de vida de gestantes infectadas pelo vírus da imunodeficiência humana (HIV) na cidade de São Paulo. Revista Brasileira de Ginecologia Obstetrícia, 2014; 36(5): 228-32. 